\title{
Subjetividad, realidad y discurso entre el determinismo estructuralista y el construccionismo social
}

\author{
SUBJECTIVITY, REALITY AND DISCOURSE, BEYOND STRUCTURALIST DETERMINISM AND SOCIAL \\ CONSTRUCTIONISM
}

Rodrigo Cornejo (rodrigo.cornejo@uchile.cl) Departamento de Psicología, Facultad de Ciencias Sociales, Universidad de Chile (Santiago, Chile) ORCID: 0000-0001-6713-0166

Natalia Albornoz (natalbmu@ug.uchile.cl) Departamento de Psicología, Facultad de Ciencias Sociales, Universidad de Chile (Santiago, Chile) ORCID: 0000-0003-3915-2442

Diego Palacios (diego.palacios@ug.uchile.cl) Departamento de Psicología, Facultad de Ciencias Sociales, Universidad de Chile (Santiago, Chile) ORCID: 0000-0002-6956-7158

\begin{abstract}
The study of the subjectivity and the subject appears today as a disputed field by different perspectives. In this article, we expose a framework to understand the subjectivity in a cultural historical approach and we propose theoretical, methodological and axiological issues for the study of subjectivity. We discuss the place of discourse in the study of social subjectivity through structuralism's positions and proposals of social constructionism. Finally, we defend that the Critical Discourse Analysis proposed by Norman Fairclough as a solid theoretical-methodological perspective for the study of the complex links between subjectivity, discourse and social reality. We propose that this approach, based on categories such as discursive practice, social practice hegemony, discursive order, intertextuality and discursive shift, can address the theoretical, methodological and axiological challenges regarding the study of subjectivity.
\end{abstract}

Key words: subjectivity, discourse, cultural historical approach, constructionism, determinism.

\section{Resumen}

El estudio de la subjetividad y del sujeto aparece en la actualidad como un campo en disputa por distintas corrientes y posiciones. Las propuestas para el estudio del campo de la subjetividad variarán de acuerdo a las concepciones teóricas y supuestos que sobre ella se tengan. En el presente artículo se plantean una serie de desafíos teóricos, metodológicos y axiológicos necesarios de afrontar para el estudio de la subjetividad. Asimismo, se discute el lugar del discurso en el estudio de la subjetividad social, debatiendo con las posiciones estructuralistas y con las propuestas del construccionismo social. Por otra parte, se expone una forma de comprender la subjetividad desde el enfoque histórico cultural y, en consistencia con lo anterior, se plantea una perspectiva teórica-metodológica pertinente para abordar las complejas relaciones entre subjetividad, discurso y realidad social: el Análisis Crítico del Discurso propuesto por Norman Fairclough. Proponemos que este enfoque, a partir de categorías como práctica discursiva, 
práctica social, hegemonía, orden discursivo, intertextualidad y cambio discursivo, permite abordar los desafíos teóricos, metodológicos y axiológicos antes planteados respecto del estudio de la subjetividad.

Palabras clave: subjetividad, discurso, histórico cultural, construccionismo, determinismo.

\section{Introducción}

En términos generales, este artículo espera aportar al debate sobre la relación entre posiciones teóricas para comprender la subjetividad y el lugar que se le da al discurso en estas propuestas. Puntualmente nos proponemos, desde un enfoque histórico cultural, debatir a nivel teórico, epistemológico, metodológico y axiológico con el tradicional determinismo estructuralista y con el emergente construccionismo social. Para cumplir este propósito organizaremos el escrito de la siguiente manera: primero, plantearemos una discusión teórica que define los aspectos constitutivos y los diversos desafíos que supone estudiar la subjetividad desde una perspectiva histórico-cultural; segundo, plantearemos algunos puntos críticos respecto al debate sobre las relaciones entre subjetividad y discurso; tercero, analizaremos la propuestas de Análisis Crítico del Discurso de Norman Fairclough como una perspectiva pertinente para el estudio de la subjetividad en el contexto del nuevo capitalismo, evitando la reificación de las prácticas discursivas que, nos parece, ha sido propia del socioconstruccionismo. Finalmente, discutiremos críticamente los aportes de la perspectiva adoptada, así como también sus limitaciones y proyecciones.

\section{La subjetividad desde una perspectiva histórico-cultural}

El estudio de la subjetividad humana constituye un campo no conquistado completamente por el conocimiento humano. Se trata de un aspecto de lo social difícil de mirar y de nombrar, pues al hacerlo nos vemos a nosotros mismos, creados socialmente y creadores a su vez de lo social. Implica asumir el desafío metodológico de volver a pensar el tema del "objeto de investigación", pero también un desafío epistemológico, teórico y ético.

Es una temática que tiene también alcances políticos, pues no hay política, ni construcción de lo social sin sujetos (individuales y colectivos). Se trata, por cierto, de un concepto en disputa, por lo que se requiere un ejercicio de distanciamiento de la racionalidad científica dominante que nos lleve a sensibilizarnos con la inestabilidad, con los movimientos de constitución de un devenir incierto construido por seres humanos que el poder pretende mostrar como cerrado, pues "el sujeto es siempre un campo problemático antes que un objeto claramente definido, pues desafía analizarlo en función de las potencialidades y modalidades de desenvolvimiento" (Zemelman 2010:2). Las diferentes concepciones sobre subjetividad tienen consecuencias para las posibilidades de acción política.

Para muchos pensadores la categoría de sujeto es el resultado más significativo de la filosofía moderna, la que se encuentra hoy en crisis. Esta idea de sujeto es tributaria del racionalismo cartesiano, que la ve referida a un principio universal de inteligibilidad, conciencia, orden, racionalidad, intencionalidad, individualidad e indivisibilidad: "La concepción de Descartes puede ser tomada como paradigmática de la interpretación clásica del sujeto que se fijó inicialmente en la filosofía moderna: el sujeto como ente autocentrado, incondicionado, omnipotente, transparente, absolutamente racional" (Acanda 2008:2).

Las propuestas de Marx y de Freud son, para muchos, las primeras que instalan una sospecha sistemática y devastadora sobre esta imagen de individuo racional en desarrollo constante. En el sistema de 
pensamiento de estos autores aparecen fuerzas sociales e inconscientes que son inmanejables por medio de la razón individual. Sin embargo, la configuración de la subjetividad como categoría de análisis no podrá ser un proceso meramente racional, de ideas y conceptos. Se trata también de un proceso histórico, pues aunque suene recursivo y paradójico, es necesario un sujeto para poder pensar la subjetividad. La concepción que tengamos de subjetividad se relacionará también con nuestra visión de realidad social y con cómo podemos pensarla. Podemos decir que hasta mediados del siglo XX este sujeto racional cartesiano seguía gozando de buena salud, al menos en el pensamiento mayoritario de la psicología y las ciencias sociales.

Nos parece que la posibilidad de constituir la subjetividad como campo de conocimiento en el pensamiento contemporáneo es un movimiento histórico que se da, no solo en la psicología, sino en distintas disciplinas de las ciencias sociales, la filosofía, la historia, la educación, la lingüística y la epistemología.

Se trata de un movimiento histórico de conocimiento que, si bien tiene antecedentes notables en los siglos XIX y comienzos del XX en autores como Marx, Freud, Gramsci y Vigotsky, toma fuerza en el último tercio del siglo XX con los aportes de pensadores como Castoriadis, Loureau, Foucault, Freire, Guattari, Deleuze, Negri, Thompson y la historia social, así como en las teorías socialmente comprometidas de América Latina expresadas en autores como Zemelman, González Rey o Marín.

Pareciera, entonces, que actualmente existen condiciones históricas para pensar el sujeto desde una perspectiva histórico-cultural. Para González Rey (2002), estas condiciones serían básicamente el desmoronamiento de los tipos de orden de pensamiento que caracterizaron a la modernidad. Sostenemos que se trata también de la emergencia de las sociedades del control (siguiendo a Deleuze), de un capitalismo cognitivo que produce subjetividad como acumulación y que, con el pasar de los años, hace posible la emergencia de una subjetividad que puede, desde aproximaciones parciales, pensarlo y así pensarse.

Dentro del amplio debate que existe actualmente sobre la subjetividad desde una perspectiva históricocultural nos parece importante relevar cuatro elementos que nos ayudan a pensar cómo estudiarla.

En primer lugar, el hecho de que el estudio de la subjetividad debe enfrentar las dicotomías históricas del pensamiento occidental "como lo social-individual, lo interno-externo, lo afectivo-cognitivo, lo intrapsíquico-interactivo" (González Rey 2000:19).

La dicotomía entre los planos individual-colectivo es un desafío necesario para pensar la subjetividad, y como decíamos antes, nos debe llevar necesariamente a repensar los vínculos entre sujeto y objeto. Para Zemelman, una de las principales dificultades en el estudio de las subjetividades es evitar los dos grandes reduccionismos de las ciencias sociales modernas: el psicologicismo individual que no considera los aspectos históricos y contextuales de los procesos de construcción de sentidos y el estructuralismo social, que reduce toda formación subjetiva a la mera expresión de procesos macro-históricos. La óptica de la singularidad contextualizada debe reemplazar la visión clásica de un individuo rodeado por un medio social.

En segundo lugar, derivado de lo anterior, las perspectivas histórico-culturales se plantean críticamente respecto a la lectura determinista, economicista y esencialista que sustentaron algunas versiones del 
marxismo muy popularizadas durante el siglo XX, como puntualizó Gramsci: "El postulado esencial del materialismo histórico, que asevera que toda fluctuación política e ideológica puede ser presentada y expuesta como expresión inmediata de la estructura (es decir, de la base) debe ser considerado en teoría como infantilismo primitivo, y combatido en la práctica con el auténtico testimonio de Marx" (Gramsci 2005:87).

Asimismo, más allá del rescate que varios autores, como Guattari o Castoriadis, hacen de las dinámicas del inconsciente, se manifiestan críticos de la creencia de que la subjetividad "se fabrica solo a través de los estadios psicogenéticos del psicoanálisis", así como cualquier intento de reducir los hechos sociales a mecanismos psicológicos e invariantes culturalmente (Guattari 1996:21).

En tercer lugar, la necesidad de abordar la contradicción aparente por un doble significado de la palabra sujeto. Por un lado, el sujeto como sujetado a un otro por control y dependencia, como constreñido a su propia identidad, a la conciencia y a su propio autoconocimiento (Foucault). Por otro lado, el sujeto como agente, capaz de subjetivar su experiencia y sus significados, como sujeto con capacidad de opción, que puede verse presionado por las estructuras y a la vez, resistirlas en un espacio de posibilidad de acción.

En cuarto lugar, la categoría subjetividad permite abrirse al problema del estudio de lo potencial, lo que aún no se ha constituido. Esto conlleva un rompimiento de la condición dada de un objeto, por medio de destacar lo procesual de lo estructurado, mediante el énfasis de su potencialidad. Zemelman lo plantea de la siguiente forma: "si el conocimiento social se plantea la necesidad de viabilizar opciones para dar una nueva dirección a la historia, es preciso reconocer, también, las potencialidades que se contienen de prácticas utopías y proyectos (...) lo anterior implica reemplazar la relación presente-pasado (fundamento del conocimiento explicativo) por la relación presente futuro (propia del quehacer político)" (Zemelman 1992b:12).

Se trata, en otras palabras, de pensar lo indeterminado, lo inédito pero viable como desafío gnoseológico. Mirar los procesos subjetivos no solo cuando ya se han objetivado, sino también y con todos los resguardos del caso cuando se están constituyendo.

\section{El desafío de estudiar de la subjetividad}

Las perspectivas histórico-culturales plantean, con diversidad, desafíos epistemológicos, teóricos, metodológicos y axiológicos para el estudio y la comprensión de la subjetividad. Según Zemelman (2010), el problema de la subjetividad está íntimamente relacionado con cuestiones básicas del conocimiento social, por lo tanto, para estudiarla es necesario partir por problematizar lo que se entiende por realidad sociohistórica. Ésta se concibe como una construcción humana, como tensión dinámica de múltiples proyectos posibles de materializarse. No es una condición externa al ser humano, no existe por sí misma con independencia del sujeto que la vive, sino que es una construcción de sentidos.

El ejercicio de pensar la subjetividad, así como lo social, requiere de una reconceptualización de la racionalidad científica en varios de sus protocolos más conocidos como la neutralidad, la objetividad o las conexiones con lo empírico. Guattari incluso plantea la idea de un cambio de paradigma: "He propuesto el concepto de intensidad ontológica. Este concepto implica un compromiso ético - estético del agenciamiento enunciativo, tanto en registros actuales como virtuales" (Guattari 1996:43). 
El acercamiento a la realidad social se da en un ejercicio que es, a la vez, metodológico, epistemológico, teórico y también axiológico, pues el proyecto y las visiones de futuro no pueden estar ausentes si tratamos de conocer una realidad que es construcción. Para Zemelman (1992a), las conexiones de lo empírico estarían delimitadas por la potencia de lo objetivo y la voluntad social que requiere dar direccionalidad a los procesos

En ese sentido, la ciencia y el conocimiento no pueden ser neutrales, pues su naturaleza es objetivasubjetiva (Freire). Los desafíos del conocimiento, entonces, no implican un desarrollo exclusivamente racional, ya que el conocimiento se construiría desde todas las facultades humanas, incluidas el sentimiento, la imaginación y la voluntad. Por lo tanto, el estudio de las subjetividades siempre será abordado por el investigador desde su subjetividad, y al interior de ella ocupan un lugar central sus propios posicionamientos respecto a la realidad social en la que se inserta, sean ellos conscientes o no.

Esto nos lleva al tema de la implicación del investigador, quien al situarse como sujeto en el acto de investigar nos hace cuestionar el tradicional análisis centrado en la relación sujeto-objeto. Esta lógica, con pretensiones de universalidad, se sustenta en una idea de objetividad externa que podría ser apropiada por el sujeto conocedor a través de un corpus teórico permanente (Zemelman 2010). En palabras de Marín (1990), la distancia entre sujeto y objeto, permite el encubrimiento de la materialidad de las relaciones sociales. Dicho de otro modo, esa lógica dicotómica muy propia de la modernidad llevaría a la complicidad del sujeto conocedor con una realidad que se muestra como objetiva e inamovible.

Igualmente, si el análisis de la realidad social y la subjetividad deben incorporar la dimensión de lo constituyente, entonces la objetividad como algo estable y determinado, tal como la conocemos en el método científico, pierde sentido. Captar la subjetividad en su dimensión de creatividad procesual, implica una elección ética a favor de la riqueza de lo posible, implica entender la realidad en su potencialidad y con la intención de construir desde lo dado y lo dándose (Zemelman 1997).

Para considerar lo constituyente, es necesario sensibilizarse hacia la inestabilidad y los movimientos de constitución de un devenir incierto, construido por seres humanos, pese a que el poder lo muestre como cerrado, estático y natural. De este modo, estudiar la subjetividad implica entender la realidad en movimiento, donde el pensamiento debe "producir y organizar el mundo en la forma diferenciada y única que se presenta al investigador en un momento dado" (González Rey 2002:87).

Para este autor la investigación de la subjetividad es un proceso básicamente constructivo-interpretativo. La cualidad de los fenómenos no aparece de forma inmediata a la experiencia, ni se puede construir totalmente por vía inductiva, lo que invita a superar la descripción y pasar a la construcción, que exprese la emocionalidad, la omisión y los énfasis necesarios (González Rey 2005).

El investigador integra y reconstruye, en construcciones interpretativas, diversos indicadores, que no tendrían sentido como constataciones empíricas aisladas. Lo descriptivo suele ser un momento necesario de la construcción interpretativa, lo cual reivindica el carácter activo del investigador en el proceso investigativo (González Rey 2007, 2013). En esta perspectiva, no tiene sentido limitarse a trabajar con estructuras teóricas acabadas frente a una mutabilidad real imprevisible y una praxis que la moldea. El significado de los contenidos no puede circunscribirse a una estructura teórica fija y determinada, ya que consideraría solo los contenidos de los universos de observación ya previstos. Por lo tanto, el desafío teórico requiere creatividad, flexibilidad y posicionamiento. 
Por ello nos planteamos la pregunta acerca de cómo estudiar la subjetividad, qué conocimiento podemos construir de ella, si el sujeto no es un ente indivisible, individual y transparente de la filosofía clásica. Pensamos que habrá que renunciar a diversas pretensiones: los universalismos de las modelizaciones psicológicas; los discursos coherentes, cerrados y sin negaciones; y la pretensión de unidad y permanencia del sujeto. Por tanto, habrá que preguntarse por los agenciamientos colectivos de enunciación, por los quiebres y discontinuidades del orden discursivo hegemónico, por las expresiones individuales como nudos de relaciones sociales contingentes. Habrá que atender las señales que permitan teorizar "cómo los agenciamientos de enunciación reales pueden poner en conexión las diferentes instancias de producción de subjetividad particularmente aquellas de carácter extrapersonales e infra personales" (Guattari y Rolnik 2006:46).

\section{¿Qué lugar asignarle al discurso en el estudio de la subjetividad?}

Llegamos así a un asunto que genera controversia y debate al interior de las ciencias sociales. Se trata más bien de un debate con posturas variadas y dinámicas, que de corrientes totalmente estructuradas. Sin embargo, podríamos plantear que los argumentos centrales en pugna son aquellos que sustentan las tesis de la denominada galaxia del "construccionismo social" (Piper 2008) y de planteamientos que hemos catalogado como histórico-culturales.

Tanto las posiciones construccionistas como los enfoques histórico-culturales, comparten el grueso de las críticas que se han esbozado respecto de la visión cartesiana del sujeto y la racionalidad científica objetivista y positivista. Las posiciones construccionistas, por su parte, enfatizan la crítica a los esencialismos y determinismos del pensamiento social moderno y rechazan cualquier intento de objetividad en los procesos de construcción de conocimiento. Asimismo, las posiciones construccionistas apuntan a la disolución de la dicotomía sujeto-objeto, al necesario relativismo e historicismo del conocimiento, a la construcción social cotidiana de la realidad y al carácter relacional y significativo del proceso de investigación (Íñiguez 2003, Piper 2008, Jorquera y Ramos 2011).

Los puntos de divergencia entre ambas posturas, creemos, están dados por la negación total, que hace el socioconstruccionismo, de la existencia de la realidad más allá de las prácticas discursivas contingentes y la concepción del lenguaje y las prácticas discursivas como las únicas dinámicas constitutivas de la subjetividad (Santander 2011).

Para el enfoque histórico-cultural, si bien el conocimiento no es una representación directa ni una traducción de algo que pertenece a la realidad externa, eso no debe llevar a negarla como hacen los construccionistas. La realidad es uno de los aspectos de la configuración del conocimiento, el cual dialoga problemáticamente con ella. En ese sentido, la realidad tendría un lugar activo en la producción de conocimiento del investigador, pues lo presiona y lo contradice (González Rey 2002). En la misma línea, Castoriadis afirma que la realidad social no es comprensible como tal por ser una construcción humana, pero sí es pensable: "el hecho de que el campo histórico social jamás sea comprensible como tal, sino solamente por sus efectos, no prueba que posea una mínima realidad, sería más bien lo contrario" (Castoriadis 2010:232).

La negación de la realidad social fuera de las prácticas discursivas contingentes tiene una serie de implicancias en el conocer y en el hacer. Como ya dijimos, existe una relación dialéctica entre discurso y estructura social, esta última es "condición para" y efecto de la primera. En este marco, los discursos no 
son textos que fluyen libremente por las cabezas de las personas, o entre ellas, sino que expresan relaciones sociales que se dan dentro de un sistema social específico, en el que existen desiguales condiciones para la creación y circulación de estos discursos. Así lo afirma Fairclough (1993), para quien los discursos surgen de la práctica social, erigida sobre las estructuras sociales materiales.

Es verdad que el discurso deviene en materialidad, pero no es la única materialidad social posible. El poder existe y opera, el poder básicamente actúa, no siempre habla. Concebir el poder actuando solo por el juego del lenguaje conlleva una visión unidimensional del mismo.

Las derivaciones éticas de las posturas construccionistas hay que valorarlas sin caer en posiciones moralistas al respecto. Un camino que nos parece válido en ese sentido es preguntarnos por sus consecuencias concretas: ¿A quién le sirve esta explicación del mundo, en la cual no existen situaciones de privilegio que sean anteriores a los intercambios conversacionales contingentes? Este argumento construccionista, llevado a su extremo, termina siendo conservador: "Replegarse en un relativismo inútil, cuando se enfrentan con temas tales como los abusos de poder, los cuales requieren juicios de verdad o falsedad, es en mi opinión, un serio fracaso ético, sin importar las voces teóricas que puedan ser usadas para racionalizar dicha postura" (Fairclough 1995:36).

Igualmente, el énfasis exclusivo en el carácter constitutivo del lenguaje puede llevar a un "discursivismo simbólico desencarnado", que termina negando el sentido y, finalmente, al sujeto como tal (González Rey 2005). El lenguaje es inseparable de un sujeto que piensa, que produce no solo significaciones, sino también sentidos subjetivos y que reproduce y también resiste órdenes discursivos de determinados órdenes sociales.

Para Laclau y Mouffe (2004) dicha negación se da por una confusión en los debates sobre el sujeto y por dos problemas de distinto orden: i) el problema del carácter discursivo o prediscursivo del sujeto y ii) el problema sobre la relación entre las distintas posiciones del sujeto.

El primer problema está relacionado con la crítica, ya señalada, del sujeto como un agente racional y transparente a sí mismo, como unidad y homogeneidad entre el conjunto de sus posiciones y como origen y fundamento de las relaciones sociales. Respecto a este asunto, la posición de los autores es discursiva, pues los sujetos serían "posiciones de sujeto en el interior de una estructura discursiva" y su experiencia depende de "condiciones discursivas de posibilidad precisas" (Laclau y Mouffe 2004:156).

¿Dónde radica la confusión citada?: "Del carácter discursivo de toda posición de sujeto no se sigue nada acerca del tipo de relación que pueda existir entre dichas posiciones (...) Si toda posición de sujeto es discursiva, el análisis no puede prescindir de las formas de sobredeterminación de unas posiciones por otras... (a no ser que hagamos) un reemplazo del esencialismo de la totalidad por un esencialismo de los elementos" (Laclau y Mouffe 2004:156).

Para Zemelman, la crisis del sujeto es más bien la crisis del sujeto racionalista y unitario que hemos descrito, concebido como un actor de grandes espacios históricos (Zemelman 2006). En el contexto de crisis de dicho sujeto y en medio de un clima de derrota de proyectos sociales alternativos de la izquierda a comienzos de los 90 s, se potenciaron las posturas construccionistas radicales, asociadas a las teorías de la postmodernidad (Fairclough 1995). 
Finalmente, la negación radical del sujeto puede terminar constituyéndose en una paradoja, al negarse también aquello que es tan preciado en los enfoques construccionistas, vale decir, el carácter construido de lo social. Con esta negación paradojal, estaríamos frente a una especie de retorno a postulados estructuralistas del lenguaje. Así lo afirma Castoriadis (2010), para quien la historia existe en el lenguaje, pero éste es constituido y transformado por la historia. Ignorar esta cuestión es "eliminar la cuestión histórica por excelencia: la génesis del sentido, la producción de nuevos sistemas de significación y de significantes" (Castoriadis 2010:223).

Resulta entonces necesario alejarse de posturas que sobredimensionan la determinación social en el discurso, así como de aquellas que plantean la construcción social únicamente a partir del discurso. Mientras en las primeras el discurso aparece como mero reflejo o epifenómeno de la realidad, en las segundas, el discurso aparece como único origen de lo social.

Desde esta perspectiva se plantea el carácter activo del investigador, quien construye el conocimiento a partir de una problematización histórica y un posicionamiento axiológico que le permite recortar la realidad a estudiar y abrirse hacia lo indeterminado. En ese sentido, la perspectiva histórico-cultural y el construccionismo social comparten la necesidad de una epistemología centrada en la construcción. No obstante, para el enfoque histórico cultural esta construcción no es visible a partir de las conversaciones, sino que está mediada por el investigador y su función constructivo-interpretativa (González Rey 2007).

A partir de esta consideración, en las siguientes líneas plantearemos la pertinencia del Análisis Crítico del Discurso de Norman Fairclough como perspectiva útil para el estudio de la subjetividad en el nuevo capitalismo. En este contexto, las relaciones económicas alcanzan una nueva escala a nivel global, el conocimiento y la información adquieren un significado particular, que es producido, circulado y consumido como discursos (Fairclough 2001).

Sostenemos que esta perspectiva permite evitar la reificación de las prácticas discursivas propia del socioconstruccionismo, reconociendo que existen prácticas humanas no necesariamente discursivas y enfatizando en el carácter activo y políticamente comprometido del investigador en la descripción, interpretación y explicación de los recortes de realidad que pretende conocer.

\section{El análisis crítico del discurso de Norman Fairclough como enfoque para estudiar la subjetividad}

El discurso suele definirse, desde las perspectivas discursivas de las ciencias sociales, como un conjunto de prácticas lingüísticas que mantienen y promueven ciertas relaciones sociales (Iñiguez 2003) y como una práctica social, un "modo de acción", para actuar en el mundo y sobre otros (Fairclough 1993). Así, considerar al discurso como una práctica social, nos invita a ver los textos singulares en dominios discursivos más amplios, en instancias de poder político, social y simbólico.

El análisis crítico del discurso (en adelante ACD) no puede considerarse como una corriente homogénea, pues alberga múltiples enfoques y diversas disciplinas. Se trataría más bien de una comunidad que comparte un interés común, un objetivo político, en el sentido de evidenciar la dominación a través de los discursos y de concebir que discursivamente se pueda hacer algo para disminuirla (Iñiguez). En ese sentido, un interés permanente de los ACD apunta hacia el descubrimiento, la revelación de lo implícito, de aquello que por alguna razón permanece escondido en las relaciones discursivas. 
Los autores del ACD asumen postulados diversos que se ubican en un continuo entre los metarrelatos construccionistas y los enfoques histórico-culturales. Estos últimos suelen inspirarse en las obras de Gramsci y Foucault, y enfatizan en la relación dialéctica que existe entre el discurso y la estructura social, más que en los intercambios conversacionales contingentes como única forma de expresión de lo macro social, a diferencia de la psicología discursiva británica y el análisis conversacional (Antaki y Díaz 2003, Edwards 2003). En esta heterogeneidad de posturas, existe consenso en considerar la estructura social como condición y efecto de las prácticas discursivas (Meersohn 2005, Santander 2011). El "discurso como práctica no solo representa al mundo, sino también lo significa, constituyendo y construyendo su significado" (Fairclough 1993:48). Esta dimensión significativa del lenguaje se basa en que "la institución de la sociedad es institución del hacer social y del representar/decir social" (Castoriadis 2010:558). Para este enfoque del $A C D$, el discurso es posible solo a partir de la reconstrucción de sujetos y viceversa, sin discurso no hay sujeto posible.

Ninguna actividad humana, el lenguaje tampoco, resulta una actividad aislada del conjunto de sentidos que caracterizan el mundo histórico y social de una persona, de una institución y de una sociedad. Para Zemelman (2006), es necesario realizar un proceso de historización y contextualización del objeto de conocimiento que se está construyendo, pues esta problematización contextual sería la manera de recuperar lo potencial de la realidad.

Desde el enfoque histórico cultural, las categorías "formaciones discursivas" y "orden discursivo" propuestas por Foucault se tornan fundamentales en el planteamiento del ACD. Conviene precisar el planteamiento foucaultiano sobre estas categorías: "juego de las reglas que hacen posible, durante un periodo determinado, la aparición de objetos, objetos recortados por medidas de discriminación y de represión" (Foucault 2005:53); "prescriben lo que ha debido ponerse en relación, en una práctica discursiva, para que esta se refiera a tal o cual objeto, para que ponga en juego tal o cual enunciado, para que utilice tal o cual conjunto, para que organice tal o cual estrategia (...) una formación discursiva determina una regularidad que les es propia a unos procesos temporales; plantea el principio de articulación entre una serie de acontecimientos discursivos y otras series de acontecimientos, transformaciones, de mutaciones, de procesos" (Foucault 2005:122).

Norman Fairclough adapta el concepto orden del discurso de Foucault y lo replantea a partir de sus investigaciones en la década de los 80s: "un conjunto ordenado de prácticas discursivas asociadas con un dominio o institución social particular (ej: la conferencia, el seminario, la orientación, y la conversación informal) y los límites y relaciones entre ellas. Las prácticas discursivas pueden estar demarcadas de forma relativamente fuerte o débil -los límites pueden ser rígidos o permeables, y las prácticas discursivas pueden entrar en varios tipos de relaciones (...) de complementariedad o de oposición" (Fairclough 1995:23).

Para Fairclough es posible hablar de un orden discursivo general, propio de una sociedad en un momento histórico, y de órdenes del discurso de carácter local, que deben ser entendidos como conjuntos de prácticas discursivas relacionadas con un particular dominio social. Por otra parte, las prácticas discursivas que componen un orden del discurso pueden ser catalogarse en tres tipos: géneros, discursos y estilos (Stecher 2014). Los géneros serían "formas socialmente ratificadas de utilizar el lenguaje en conexión con un particular tipo de actividad social", los discursos serían "formas estabilizadas de representar ciertos aspectos del mundo social" y los estilos serían "formas relativamente estabilizadas de ser y actuar discursivamente" (Stecher 2010:101). A la base de esta concepción encontramos una teoría social del 
lenguaje que resulta clave para comprender la propuesta teórico-metodológica de Fairclough. Desde esta teoría, se distinguen tres efectos constitutivos del discurso, los cuales corresponden a tres funciones del lenguaje que coexisten e interactúan en todo discurso: i) función identitaria, que alude a la construcción de identidades de los sujetos; ii) función relacional, que da cuenta de cómo los sujetos construyen relaciones sociales y se posicionan respecto a otros; y iii) función ideacional, que apunta a la construcción de sistemas de creencias y conocimiento respecto del mundo (Fairclough 1993).

La práctica discursiva contribuye a la (re)producción del orden social, pero también puede ocupar un lugar en su transformación. El discurso está formado y restringido por la estructura social, en tanto repertorios disponibles, normas de uso aceptadas y "desiguales capacidades de controlar cómo los textos son producidos, distribuidos y consumidos en contextos sociales particulares" (Fairclough 1995:2). Pero por otro lado el discurso es socialmente constituyente, pues no solo representa al orden social, sino que también constituye y construye su significado (Fairclough 1993).

Se plantea una relación dialéctica entre discurso y estructura social, pues el discurso puede reflejar y a la vez construir la realidad social, en tanto la realidad social no se constituye únicamente por el discurso sino por un conjunto prácticas sociales que pueden ser o no discursivas (Fairclough 1995). Desde esta premisa, resulta necesario alejarse de posturas que sobredimensionan la determinación social en el discurso, así como la construcción de lo social, exclusivamente como producto del discurso.

Para Fairclough, el discurso como práctica política establece, sostiene y cambia las relaciones de poder y las entidades colectivas (clases, comunidades, grupos). Asimismo, el discurso como práctica ideológica, constituye, naturaliza, sostiene y cambia las significaciones del mundo, desde los diversos lugares en las relaciones de poder (Fairclough 1993). En un texto posterior, lo plantea de la siguiente manera: "El poder de controlar un discurso es visto como el poder de sustentar prácticas discursivas particulares, en un lugar de dominancia sobre otras prácticas alternativas (incluidas las que se oponen a ellas) (...) Propongo que (...) en las sociedades capitalistas modernas y contemporáneas (tardías) el discurso adquiere un rol aún más esencial en la reproducción y en el cambio sociocultural” (Fairclough 1995:3).

En este análisis de la dialéctica discurso-estructura social, Fairclough atribuye un lugar central a la categoría "hegemonía", propuesta por Gramsci, para comprender las condiciones de producción del discurso desde una mirada dinámica. Esta categoría es dinámica, da cuenta de una tensión y, por ello, solo es posible de pensar como tal en relación a otros términos. Se trata de una categoría central en el pensamiento crítico contemporáneo, que deja de pensar el poder como si estuviera "alojado" en instituciones estatales ubicadas fuera de los espacios cotidianos e intersubjetivos.

Con la categoría hegemonía, Gramsci sitúa la mirada en la construcción histórica, a través de la sociedad civil, de todo un sistema de valores, creencias, moralidad, concepciones de la realidad que, aun siendo expresión de la visión de mundo de alianzas de grupos sociales concretos -lo que él llamó un bloque histórico-, se muestran con apariencia de universalidad. Se plantea así la necesidad de poner atención sobre los procesos de construcción del sentido común de una época, que aparecen como naturales, ya dados y que, por lo mismo, son difíciles de apreciar.

Volviendo a Fairclough, su planteamiento recoge la categoría de hegemonía, pues permite analizar cómo las relaciones de poder restringen y controlan la productividad y la creatividad en las prácticas discursivas. De igual modo, esta categoría entrega una herramienta conceptual para observar los procesos de 
constitución y cambio en las prácticas discursivas y sociales.

Específicamente, para Fairclough (1993) un dominio hegemónico sería una configuración histórica de prácticas discursivas relativamente estables. Desde la mirada gramsciana, la ideología, que ha ocupado un lugar destacado en los debates sobre análisis del discurso, es parte del entramado hegemónico del bloque histórico en el poder. En otras palabras, ideología es una concepción del mundo que se va expresando en los distintos dominios de la vida social (arte, leyes, actividades económicas) y también en la vida individual de las personas.

Fairclough también rescata lo que él considera una concepción innovadora de Gramsci respecto de la relación entre sujeto y hegemonía. Un aspecto de la construcción de hegemonía consiste en constituir sujetos capaces de interpretar el discurso hegemónico, para quienes sus conexiones sean naturales y obvias: "en Gramsci aparece una concepción del sujeto como estructurado por diversas ideologías implícitas en sus prácticas que, en conjunto, le dan un carácter extrañamente complejo y una concepción del sentido común como una reserva de diversos efectos de las luchas ideológicas pasadas y un constante blanco para reestructurar las luchas por venir. En el sentido común, la ideología se vuelve naturalizada. Por otra parte, Gramsci entiende el terreno de las ideologías en términos de conflictos superpuestos, intersectados o en formación y los denomina complejo ideológico" (Fairclough 1993:71).

De esta manera, la concepción dialéctica y dinámica de lucha hegemónica propuesta por Gramsci resulta coherente con la propuesta metodológica planteada por Fairclough, la que pone énfasis en las relaciones entre estructuras discursivas y eventos sociales: "Un orden del discurso puede verse como la faceta discursiva del equilibrio contradictorio e inestable que constituye la hegemonía y, por otro lado, la articulación y rearticulación de los órdenes del discurso constituyen un pilar fundamental de la lucha hegemónica. Es más, la práctica discursiva, la producción, distribución y consumo (e interpretación) de los textos constituyen una faceta de la lucha hegemónica que contribuye en diversos grados a la reproducción o transformación, no solo del orden del discurso existente, sino también de la vida social y de las relaciones de poder" (Fairclough 1993:71).

Por ello, la propuesta metodológica de este autor busca trazar una conexión explicativa entre instancias particulares del discurso, prácticas discursivas y sociales, en una propuesta en la que se funde el análisis del discurso y el análisis social: "Hacer aparecer en su pureza el espacio en que se despliegan los acontecimientos discursivos no es tratar de restablecerlo en un aislamiento que no se podría superar; no es encerrarlo sobre sí mismo, es hacerse libre para describir en él y fuera de él juegos de relaciones" (Fairclough 1993:47).

Si bien la tensión que intenta articular Fairclough es entre la práctica discursiva (como evento) y la práctica social (como estructura social construida), nos parece útil para estudiar las articulaciones entre sujetos, discursos y estructuras sociales. Entre otras cosas porque para este autor las operaciones discursivas son producidas por sujetos sociales, tal como se puede apreciar en el concepto de "tecnologización del discurso", propuesto para dar cuenta de "intervenciones calculadas" para el desplazamiento de prácticas discursivas como parte de la ingeniería del cambio social (Fairclough 1993). Esto nos parece particularmente importante de considerar en el momento histórico presente, donde la sociedad como modo de producción de vida es refundada desde sus raíces mismas por el poder, y tanto el lenguaje como la subjetividad ocupan un lugar central en dicho proceso de refundación. 
Finalmente, la categoría de "cambio discursivo" nos resulta particularmente relevante. Fairclough incorpora los aportes de Bakhtin sobre la interdiscursividad y la intertextualidad. Por una parte, la interdiscursividad es una configuración de formaciones discursivas interdependientes que subyace al evento discursivo y que no es predecible sólo a partir de un texto particular.

Por otra parte, la intertextualidad remite a la localización del texto con respecto a la red social de órdenes del discurso, cómo se actualiza y cómo entiende su potencial dentro de esta red. Para Fairclough, la intertextualidad inherente a los discursos y la historicidad de la producción y la interpretación textual, configura la creatividad como una opción y el cambio discursivo como algo constante: "El cambio (discursivo) involucra formas de trasgresión: nuevas combinaciones a partir de las combinaciones existentes o el uso de convenciones en situaciones en las que comúnmente estaban excluidas (...) el cambio deja trazas bajo la forma de co-ocurrencias de elementos contradictorios o inconscientes (mezclas de estilos formales e informales, vocabularios técnicos y no técnicos, marcas de autoridad y familiaridad, formas sintácticas típicas de la escritura y de la oralidad, etc.)" (Fairclough 1993:74).

La heterogeneidad de los textos sería un indicador sensible de cambio discursivo y de las contradicciones y cambios socioculturales. Esta heterogeneidad es posible ya que todo texto es, en parte repetición, y en parte creación, es un espacio de tensión entre fuerzas centrípetas y centrífugas del lenguaje que se expresan en una matriz de relaciones de poder (Fairclough 1993).

En la propuesta de Guattari (1996), también encontramos un énfasis metodológico en la aprehensión de lo que él denomina "desvíos de la discursividad", los que comprende como enunciados parciales y segmentos semióticos que adquieren autonomía, se ponen a trabajar por su "cuenta propia" y de esa forma pueden generar nuevos campos de referencia.

Del mismo modo, Fairclough sostiene que la valoración concreta del cambio discursivo solo es posible a través de la mirada acerca de la relación entre el evento discursivo concreto, las prácticas discursivas y la estructura social. En ese sentido, Fairclough rescata la siguiente cita de Foucault: "No existe el discurso del poder por un lado y, enfrente, otro que se le oponga. Los discursos son elementos tácticos o bloques de operación en el campo de las relaciones de fuerza; puede haberlos diferentes e incluso contradictorios en el interior de la misma estrategia; pueden por el contrario circular sin cambiar de forma entre estrategias opuestas" (Foucault 2005:124).

El estudio del cambio discursivo se nos presenta como una estrategia posible de análisis para intentar dar cuenta, no solo del discurso dado, sino también de lo que "está dándose" en el discurso subjetivo, de los procesos de constitución discursiva, previos al momento de su materialización y realización. Este ejercicio es posible a partir de una teoría social que deja de concebir al sujeto como inevitable punto de llegada de un proceso de organización social.

\section{Discusión y Conclusiones}

La perspectiva histórico-cultural aquí desarrollada se presenta como opción pertinente para el estudio de las complejas relaciones entre subjetividad, realidad y discurso. A su vez, se configura como una opción teórica, epistemológica, metodológica y axiológica que permite poner en tensión la tradición del determinismo estructural y el emergente construccionismo social. Particularmente en discusión con este enfoque, que por su carácter seductor se ha instalado como la tendencia predominante en psicología, 
presentamos una conceptualización de subjetividad que no fuera considerada meramente como resultado de procesos macro-históricos o como una producción discursiva situada que ignora la materialidad sobre la cual se produce.

Al respecto, las palabras de González Rey sintetizan con fuerza nuestra posición: "La subjetividad se desarrolla en las prácticas culturales sobre las que se organiza la vida social, pero es irreducible a las representaciones y creencias del hombre. Por otra parte, la noción de subjetividad también se opone al reduccionismo cultural que se representa al hombre como un mero epifenómeno de sus prácticas discursivas. La reducción lingüístico - discursivo - relacional usada por el construccionismo social para superar el individualismo metafísico de la psicología, representa una versión sutil de racionalismo, pues reduce la acción humana a producciones sociales, desconsiderando los diversos procesos de naturaleza diferente que tienen capacidad generadora sobre la acción humana" (2013:34).

En línea con dicha consideración teórica y epistemológica es que el Análisis Crítico del Discurso desarrollado por Norman Fairclough representa, en nuestra opinión, una perspectiva metodológicopráctica y axiológica pertinente para estudiar un recorte de realidad a partir del discurso sin que esto signifique ontologizar lo discursivo. Este enfoque, a través de la puesta en juego de categorías como práctica discursiva, práctica social, orden discursivo, hegemonía, inter textualidad y cambio discursivo permite abordar complejamente las dicotomías individual-social e intrapsíquico-interactivo, superar las lecturas deterministas de la estructura social, leer la doble dimensión de los sujetos (en su sujeción y agenciamiento) y abrirse a lo indeterminado y la potencialidad del cambio discursivo.

Lo anterior implica reconocer un asunto metodológico-práctico que opera en una doble vía: junto con ser necesario plantear dispositivos que presenten los procesos investigativos en ciencias sociales como instancias dialógicas entre sujetos que comparten un tiempo y un espacio, es también preciso desarrollar exploraciones que no se reduzcan a la producción situada de discursos mediante el diálogo. En ese sentido, desde enfoques interpretativos es esperable un desarrollo cada vez más complejo e integrado de diseños que articulen el orden del decir y el orden del hacer, desde la interpretación dialógica y la coproducción de los datos.

Finalmente, consideramos que los argumentos expuestos se presentan como buenas razones para reivindicar el carácter activo y socialmente comprometido del investigador posicionado desde una perspectiva histórico-cultural. En una era caracterizada por la carencia de palabras apropiadas para nombrar incluso lo cotidiano, se precisa recuperar el carácter creativo y subversivo de la subjetividad a fin de crear nuevos horizontes de posibilidades que permitan pensar la realidad social desde los sujetos, aunque estos aparezcan ocultos en el curso de la historia. Esta tarea no representa un mero ejercicio intelectual, sino una invitación para reivindicar la especulación como forma de conocimiento, la actividad científica como una tarea constructivo-interpretativa y, en palabras de Zemelman: "la esperanza en su función forjadora de la subjetividad (...) Se requiere de un conocimiento que facilite a quien lo construye y a quien lo utilice darse cuenta de lo que significa ser sujeto: en suma, que contribuya al desarrollo de su conciencia como protagonista de la historia, por lo tanto constructor de las circunstancias que conforman el espacio de su destino" (2005:26).

Esperamos con este artículo haber aportado al debate sobre el estudio de las complejas relaciones entre subjetividad, realidad y discurso. Debate que no es posible ni deseable dar por cerrado, pero en el que nos 
corresponde buscar los mayores niveles posibles de consistencia teórica, epistemológica, metodológica y axiológica.

\section{Agradecimientos}

Este artículo se pudo realizar gracias al apoyo económico de Consejo Nacional de Ciencia y Tecnología (Chile), a través de su proyecto FONDECYT N${ }^{\circ} 11130244$.

\section{Bibliografía}

Acanda, L. 2008. La problemática del sujeto y los desafíos para la teoría de la educación. Blog post http://www.rebelion.org/noticia.php?id=69633

Antaki, Ch. y Díaz, F. 2003. El análisis de la conversación y el estudio de la interacción social. En: L. Iñiguez (ed.). Análisis del discurso. Manual para las ciencias sociales (pp. 125-140). Barcelona: UOC

Castoriadis, C. 2010. La institución imaginaria de la sociedad. Buenos Aires: Tusquets.

Edwards, D. 2003. Psicología discursiva: el enlace entre la teoría y el método a través de un ejemplo. En: L. Iñiguez (ed.) Análisis del discurso. Manual para las ciencias sociales (pp. 141-156). Barcelona: UOC.

Fairclough, N. 2001. El análisis crítico del discurso como método para la investigación en ciencias sociales. En R. Wodak y M. Meyer (comps.) Métodos de análisis crítico del discurso (pp. 179-201). Barcelona: Gedisa.

Fairclough, N. 1995. Critical discourse analysis. The critical study of language. London: Longman.

Fairclough, N. 1993. Discourse and social change. Cambridge: Polity Press.

Foucault, M. 2005. La arqueología del saber. Buenos Aires: Siglo XXI.

González Rey, F. 2013. Subjetividad, cultura e investigación cualitativa en psicología: la ciencia como producción culturalmente situada. Liminales 1(4): 13-36.

González Rey, F. 2007. Investigación cualitativa y Subjetividad. Los procesos de construcción de información. México: McGraw Hill.

González Rey, F. 2005. Subjetividad: una perspectiva histórico cultural. Universitas Psychologica 4(3): 373-383.

González Rey, F. 2002. Sujeto y subjetividad. Una aproximación histórico cultural. México: Thompson. González Rey, F. 2000. Investigación cualitativa en psicología. Rumbos y desafíos. México: Thompson. Gramsci, A. 2005. Antología. México: Siglo XXI.

Guattari, F. y Rolnik, S. 2006. Micropolítica. Cartografías del deseo. Traficantes de sueños: Madrid. Guattari, F. 1996. Caosmosis. Hacia un nuevo paradigma estético. Buenos aires: Manantial.

Iñiguez, L. 2003. Análisis del discurso. Manual para las ciencias sociales. Barcelona: Editorial UOC.

Jorquera, T. y Ramos, L. 2011. De la casa de las campanas a la máquina del tiempo. Memoria para optar al Título de Psicóloga. Santiago: Universidad de Chile. 
Laclau, E. y Mouffe, C. 2004. Hegemonía y estrategia socialista. Hacia una radicalización de la democracia. Buenos Aires: Fondo de Cultura Económica.

Marín, J.C. 1990. Conversaciones sobre el poder: una experiencia colectiva. Buenos Aires: Instituto de Investigaciones Gino Germani.

Meersohn, C. 2005. Introducción a Teun van Dijk: análisis de discurso. Cinta Moebio 24: 288-302. www.moebio.uchile.cl/24/meersohn.htm

Piper, I. 2008. Socio construccionismo y sus usos en psicología. En: A. Kaulino y A. Stecher (ed.). Materiales para una cartografía de la psicología contemporánea: Tradiciones teóricas (pp.337-348). Santiago: Serie Universitaria LOM.

Santander, P. 2011. Por qué y cómo hacer análisis de discurso. Cinta moebio 41:207-224 doi: http://dx.doi.org/10.4067/S0717-554X2011000200006

Stecher, A. 2010. El análisis crítico del discurso como herramienta de investigación psicosocial del mundo del trabajo. Discusiones desde América Latina. Universitas Psychologica 9(1): 93-107.

Zemelman, H. 2010. Sujeto y subjetividad: la problemática de las alternativas como construcción posible. Polis 27: 2-10. http://polis.revues.org/943

Zemelman, H. 2006. El conocimiento como desafío posible. México: IPN.

Zemelman, H. 2005. Voluntad de conocer. El sujeto y su pensamiento en el paradigma crítico. Barcelona: Anthropos.

Zemelman, H. 1997. Subjetividad: umbrales del pensamiento social. México: Anthropos.

Zemelman, H. 1992 a. Los horizontes de la razón. Uso crítico de la teoría. México: Anthropos.

Zemelman, H. 1992 b. Educación como construcción de sujetos sociales. Revista La Piragua 5: 12-18.

Recibido el 13 Abr 2016

Aceptado el 16 May 2016 